PROCEEDINGS OF THE

AMERICAN MATHEMATICAL SOCIETY

Volume 128, Number 12, Pages 3693-3699

S 0002-9939(00)05461-7

Article electronically published on May 18, 2000

\title{
FOUR-GENUS AND FOUR-DIMENSIONAL CLASP NUMBER OF A KNOT
}

\author{
HITOSHI MURAKAMI AND AKIRA YASUHARA
}

(Communicated by Ronald A. Fintushel)

\begin{abstract}
For a knot $K$ in the 3 -sphere, by using the linking form on the first homology group of the double branched cover of the 3-sphere, we investigate some numerical invariants, 4-genus $g^{*}(K)$, nonorientable 4-genus $\gamma^{*}(K)$ and 4dimensional clasp number $c^{*}(K)$, defined from the four-dimensional viewpoint. T. Shibuya gave an inequality $g^{*}(K) \leq c^{*}(K)$, and asked whether the equality holds or not. From our result in this paper, we find that the equality does not hold in general.
\end{abstract}

\section{INTRODUCTION}

We shall work in piecewise linear and locally flat category. All 4-manifolds and 3-manifolds will be assumed to be oriented.

In [16] T. Shibuya introduced some numerical invariants for classical links from the four-dimensional viewpoint. In this paper, we restrict ourselves to knots and study the relation between the 4-genus (slice genus) and the 4-dimensional clasp number. We also introduce another four-dimensional numerical invariant, the nonorientable 4-genus, for knots.

The 4-genus $g^{*}(K)$ of a knot $K$ in $S^{3}=\partial B^{4}$ is the minimum genus of orientable surfaces in $B^{4}$ bounded by $K[5]$. The nonorientable 4-genus $\gamma^{*}(K)$ is the minimum first Betti number of nonorientable surfaces in $B^{4}$ bounded by $K$. For a slice knot, it is defined to be 0 instead of 1 . Shibuya [16] defined the 4-dimensional clasp number $c^{*}(K)$ to be the minimum number of the double points of transversely immersed 2-disks in $B^{4}$ bounded by $K$.

Shibuya 16 gave the following inequality

$$
g^{*}(K) \leq c^{*}(K)
$$

and asked whether the equality holds or not. Note that since $g^{*}\left(3_{1}\right)=c^{*}\left(3_{1}\right)=1$, the equality above is best possible. In section 11 we investigate the 4-dimensional clasp number and show that the equality above does not hold in general. For example, we prove $c^{*}\left(8_{16}\right)=2$ and $g^{*}\left(8_{16}\right)=1$ (Example 1.5). Here we use the notation of J.W. Alexander and G.B. Briggs [2]. See also [3], [14].

Received by the editors September 29, 1998 and, in revised form, January 29, 1999.

2000 Mathematics Subject Classification. Primary 57M25.

Key words and phrases. 4-genus, 4-dimensional clasp number, linking form.

The first author's research was partially supported by Waseda University Grant for Special Research Projects (\#98A-623) and Grant-in-Aid for Scientific Research (C) (\#09640135), the Ministry of Education, Science, Sports and Culture. 
In section 22 we investigate relations among $\gamma^{*}(K), g^{*}(K)$ and $c^{*}(K)$, and give some upper bounds for $\gamma^{*}(K)$ in terms of $g^{*}(K)$ or $c^{*}(K)$. We also investigate $\gamma^{*}(K)$ and give a necessary condition for a knot whose nonorientable 4-genus is $n$.

\section{FOUR-DIMENSIONAL CLASP NUMBER}

From now on, for a manifold $M$ and a submanifold $N$ of $M$ with codimension two, $D_{N}(M)$ denotes the double branched cover of $M$ with branched set $N$.

For an oriented 3-manifold $M$ with finite first homology group, the linking form

$$
\lambda: H_{1}(M ; \mathbb{Z}) \times H_{1}(M ; \mathbb{Z}) \longrightarrow \mathbb{Q} / \mathbb{Z}
$$

is defined as follows. Let $x$ and $y$ be 1-cycles in $M$. Suppose that $n x$ bounds a 2-chain $c$ for some $n \in \mathbb{Z}$. Then

$$
\lambda([x],[y])=\frac{c \cdot y}{n} \in \mathbb{Q} / \mathbb{Z},
$$

where $c \cdot y$ is the intersection number of $c$ and $y$.

Let $K$ be a knot in $S^{3}$ and $D_{K}\left(S^{3}\right)$ the double branched cover of $S^{3}$ with branched set $K$. Then a Goeritz matrix $U$ [7 for $K$ is a relation matrix for $H_{1}\left(D_{K}\left(S^{3}\right) ; \mathbb{Z}\right)$, and the linking form on $H_{1}\left(D_{K}\left(S^{3}\right) ; \mathbb{Z}\right.$ ) is given by $\pm U^{-1}$ (the sign depending on the choice of orientation of $\left.D_{K}\left(S^{3}\right)\right)$ [15], 8].

In this section, we prove the following theorem.

Theorem 1.1. Let $K$ be a knot in $S^{3}$ with $c^{*}(K)=1$, and let $D_{K}\left(S^{3}\right)$ be the double branched cover of $S^{3}$ with branch set $K$. Then the linking form $\lambda$ on $H_{1}\left(D_{K}\left(S^{3}\right) ; \mathbb{Z}\right)$ splits into a direct sum $\left(G_{1}, \lambda_{1}\right) \oplus\left(G_{2}, \lambda_{2}\right)$ such that

1. there is an element $g \in G_{1}$ with $\lambda_{1}(g, g)= \pm 2 /\left|G_{1}\right|$, and

2. there is a subgroup $H$ of $G_{2}$ such that $|H|^{2}=\left|G_{2}\right|$ and $\lambda_{2}\left(h, h^{\prime}\right)=0$ for any $h, h^{\prime} \in H$.

In the theorem above, $\lambda_{2}$ is called metabolic.

Remark 1.2. We can regard the theorem above as a 4-dimensional version of a result of W.B.R. Lickorish on the unknotting number 9], which states that if the unknotting number of a knot $K$ is 1 , then the linking form $\lambda$ on $H_{1}\left(D_{K}\left(S^{3}\right)\right.$; $\left.\mathbb{Z}\right)$ is of the form as $\lambda_{1}$ in Theorem 1.1. Here we need $\lambda_{2}$ as a '4-dimensional part'.

In order to prove Theorem 1.1, we need the following two lemmas.

Lemma 1.3 (Gilmer [6, Lemma 1]). Let $M$ be a rational homology 3-sphere and $\lambda$ the linking form on $H_{1}(M ; \mathbb{Z})$. If $M$ bounds a 4 -manifold $W$ with an intersection matrix $V$ on $H_{2}(W ; \mathbb{Z})$, then $\lambda$ splits into a direct sum $\left(G_{1}, \lambda_{1}\right) \oplus\left(G_{2}, \lambda_{2}\right)$ such that

1. $\operatorname{det}(V)= \pm\left|G_{1}\right|$ and $\lambda_{1}$ is represented by the matrix $-V^{-1}$, and

2. $\lambda_{2}$ is metabolic.

Here $\lambda_{1}$ is said to be represented by $-V^{-1}$ if there are some generators $u_{1}, u_{2}, \ldots, u_{n}$ of $G_{1}$ such that $\lambda_{1}\left(u_{i}, u_{j}\right)$ is equal to the $(i, j)$-entry of $-V^{-1}$ for any $i, j$.

Lemma 1.4. Let $W$ be a compact 4-manifold with $H_{1}(W ; \mathbb{Z})=0$ and $\partial W \cong S^{3}$, and let $F$ be a properly embedded, compact, possibly nonorientable surface in $W$ with $\partial F \cong S^{1}$. If $F$ represents zero in $H_{2}\left(W, \partial W ; \mathbb{Z}_{2}\right)$, then $\beta_{2}\left(D_{F}(W)\right)=2 \beta_{2}(W)+$ $\beta_{1}(F)$, where $\beta_{i}$ is the $i$-th rational Betti number. 
Proof. Let $\left(W^{\prime}, F^{\prime}\right)$ be the pair of the closed 4-manifold and the closed surface that is obtained from two copies of $(W, F)$ by gluing their common boundaries. Note that $F^{\prime}$ represents zero in $H_{2}\left(W^{\prime} ; \mathbb{Z}_{2}\right)$. It follows from [13] 3.2 and 7.2] that $\beta_{2}\left(D_{F^{\prime}}\left(W^{\prime}\right)\right)=4 \beta_{2}(W)+2 \beta_{1}(F)$. Since $\partial\left(D_{F}(W)\right)$ is a rational homology 3 -sphere, from the Mayer-Vietris exact sequence we have $\beta_{2}\left(D_{F^{\prime}}\left(W^{\prime}\right)\right)=2 \beta_{2}\left(D_{F}(W)\right)$. Hence we have $\beta_{2}\left(D_{F}(W)\right)=2 \beta_{2}(W)+\beta_{1}(F)$.

Proof of Theorem 1.1] Suppose that $c^{*}(K)=1$. Then there is an immersed 2-disk in a 4-ball with exactly one double point and with boundary $K$. By removing an open, small neighborhood of the double point, we obtain a disk with two holes $Y$ properly embedded in $S^{3} \times I$ such that $Y \cap\left(S^{3} \times\{0\}\right)=K$ and $Y \cap\left(S^{3} \times\{1\}\right)$ is a Hopf link $L$. Since a Hopf link bounds an annulus $A$ in the boundary of a 4-ball $B_{0}^{4}$, we have an orientable surface $F=Y \cup A$ with genus 1 in the 4ball $B^{4}=\left(S^{3} \times I\right) \cup B_{0}^{4}$. Thus we see $D_{F}\left(B^{4}\right)=D_{Y}\left(S^{3} \times I\right) \cup D_{A}\left(B_{0}^{4}\right)$ with $\partial\left(D_{F}\left(B^{4}\right)\right)=D_{K}\left(S^{3}\right)$. Note that $\partial\left(D_{Y}\left(S^{3} \times I\right)\right)=D_{K}\left(S^{3}\right) \cup \mathbb{R} P^{3}$ since $D_{L}\left(S^{3}\right)$ is the 3 -dimensional real projective space $\mathbb{R} P^{3}$. We may assume that $D_{A}\left(B_{0}^{4}\right)$ is a 4-ball with 2-handle $h^{2}$ attached along the trivial knot in its boundary with framing \pm 2 according to the linking number of $L$ (for example, see [1]). By Lemma 1.4 we have that $\beta_{2}\left(D_{F}\left(B^{4}\right)\right)=2$. Hence there is an intersection matrix $V$ with respect to some basis $\alpha_{1}$ and $\alpha_{2}$ of $H_{2}\left(D_{F}\left(B^{4}\right) ; \mathbb{Z}\right) /($ torsion part). Set

$$
V=\left(\begin{array}{cc}
a & c \\
c & b
\end{array}\right) .
$$

Lemma 1.3 states that the linking form $\lambda$ splits into a direct $\operatorname{sum}\left(G_{1}, \lambda_{1}\right) \oplus\left(G_{2}, \lambda_{2}\right)$ such that

1. $a b-c^{2}= \pm\left|G_{1}\right|$ and $\lambda_{1}$ is represented by the matrix $-V^{-1}$, and

2. $\lambda_{2}$ is metabolic.

Now we will find an element $g \in G_{1}$ with $\lambda_{1}(g, g)= \pm 2 /\left|G_{1}\right|$. Let $\beta$ be an element in $H_{2}\left(D_{A}\left(B_{0}^{4}\right) ; \mathbb{Z}\right)$ represented by a 2 -sphere that consists of the core of 2 -handle $h^{2}$ and a 2-disk bounded by attaching a sphere of $h^{2}$. Note that the self-intersection number is \pm 2 . From the Mayer-Vietoris exact sequence we have the natural injection $j: H_{2}\left(D_{A}\left(B_{0}^{4}\right) ; \mathbb{Z}\right) \longrightarrow H_{2}\left(D_{F}\left(B^{4}\right) ; \mathbb{Z}\right)$ since $H_{2}\left(\mathbb{R} P^{3} ; \mathbb{Z}\right)=0$. Therefore $j(\beta)$ is an element $x \alpha_{1}+y \alpha_{2}$ in $H_{2}\left(D_{F}\left(B^{4}\right) ; \mathbb{Z}\right) /\left(\right.$ torsion part) and $(x, y) V^{t}(x, y)= \pm 2$. Hence for some generators $u_{1}$ and $u_{2}$ of $G_{1}$, there is an element $g=y u_{1}-x u_{2}$ such that $\lambda_{1}(g, g)=(y,-x)\left(-V^{-1}\right)^{t}(y,-x)=\mp 2 /\left(a b-c^{2}\right)$. This completes the proof.

The following example implies that the equality of Shibuya's inequality $g^{*}(K) \leq$ $c^{*}(K)$ [16] does not hold in general.

Example 1.5. $c^{*}\left(8_{16}\right)=2$ and $g^{*}\left(8_{16}\right)=1$.

Proof. Since we can easily see that $c^{*}\left(8_{16}\right) \leq 2$ and $g^{*}\left(8_{16}\right)=1$, we shall prove that $c^{*}\left(8_{16}\right) \geq 2$. A Goeritz matrix for $8_{16}$ is

$$
\left(\begin{array}{ccc}
3 & -1 & -1 \\
-1 & 4 & -1 \\
-1 & -1 & 4
\end{array}\right) .
$$

So we see that $H_{1}\left(D_{8_{16}}\left(S^{3}\right) ; \mathbb{Z}\right) \cong \mathbb{Z} / 35 \mathbb{Z}$ and that the linking form $\lambda$ is defined by $\lambda(g, g)= \pm 11 / 35$ for some generator $g$. Note that $\lambda$ has no metabolic part because 35 is square free. Suppose that $c^{*}\left(8_{16}\right) \leq 1$. From Theorem 1.1, there is 
some integer $n$ such that $\lambda(n g, n g)= \pm 2 / 35$. Thus we have $\pm 2 / 35=\lambda(n g, n g)=$ $n^{2} \lambda(g, g)=11 n^{2} / 35$ in $\mathbb{Q} / \mathbb{Z}$. This implies that

$$
n^{2} \equiv 16 \times 11 n^{2} \equiv 16 \times( \pm 2) \equiv \mp 3(\bmod 35) .
$$

Hence we have $n^{2} \equiv \pm 3(\bmod 7)$ and $n^{2} \equiv \pm 3(\bmod 5)$. This is a contradiction.

\section{VARIOUS FOUR-GENERA}

The nonorientable genus $\gamma(K)$ of a knot $K$ is the minimum first Betti number of nonorientable surfaces bounded by $K$ [4, [12]. (In [4, [12] the nonorientable genus is called the crosscap number and is denoted by $C(K)$.) For the trivial knot, it is defined to be 0 instead of 1 . From the definitions, the following proposition is clear.

Proposition 2.1. For any knot $K$, the following inequality holds:

$$
\gamma^{*}(K) \leq \gamma(K)
$$

Since we can construct a nonorientable surface from an orientable surface by adding a half-twisted band, we have inequality similar to [4], [12].

Proposition 2.2. For any knot $K$, the following inequality holds:

$$
\gamma^{*}(K) \leq 2 g^{*}(K)+1
$$

For a knot $K$, we define $\Gamma^{*}(K)$ to be $\min \left\{2 g^{*}(K), \gamma^{*}(K)\right\}$. By this definition, an inequality $\Gamma^{*}(K) \leq \gamma^{*}(K)$ clearly holds. For a relation with the 4-dimensional clasp number, we have

Proposition 2.3. For any knot $K$, the following inequality holds:

1. $\Gamma^{*}(K) \leq \begin{cases}c^{*}(K) & \text { if } c^{*}(K) \text { is even, } \\ c^{*}(K)+1 & \text { otherwise. }\end{cases}$
2. $\gamma^{*}(K) \leq \begin{cases}c^{*}(K) & \text { if } c^{*}(K) \text { is even and } c^{*}(K) \neq 2, \\ c^{*}(K)+1 & \text { otherwise. }\end{cases}$

The inequalities in Propositions 2.1 and 2.3 are best possible. See Remark 2.9 (1), (2).

Proof. First we consider the case that $c^{*}(K)$ is even. Set $c^{*}(K)=2 m$. Then there is an immersed 2-disk $\Delta$ in a 4 -ball $B^{4}$ with $2 m$ double points $p_{1}, p_{2}, \ldots, p_{m}$, $q_{1}, q_{2}, \ldots, q_{m}$ and with boundary $K$, and there are mutually disjoint $\operatorname{arcs} a_{1}, a_{2}, \ldots, a_{m}$ in $\Delta$ such that each $a_{i}$ joins $p_{i}$ to $q_{i}$. Let $D$ be a domain 2-disk of $\Delta$ and $p_{i j}$ (resp. $\left.q_{i j}\right)(j=1,2)$ the preimages of $p_{i}$ (resp. $\left.q_{i}\right)(i=1,2, \ldots, m)$. We may assume that the preimage of $a_{i}$ joins $p_{i 1}$ to $q_{i 1}$. Let $D\left(p_{i}\right)$ and $D\left(q_{i}\right)$ be 2-disks in $\Delta$ that are images of small neighborhoods of $p_{i 2}$ and $q_{i 2}$ in $D$ respectively. It is not hard to see that there are mutually disjoint embeddings $h_{i}: D^{2} \times I \longrightarrow$ $B^{4}(i=1,2, \ldots, m)$ such that $h_{i}(\{0\} \times I)=a_{i}, h_{i}\left(D^{2} \times \partial I\right)=D\left(p_{i}\right) \cup D\left(q_{i}\right)$ and $h\left(D^{2} \times I\right) \cap \Delta=a_{i} \cup D\left(p_{i}\right) \cup D\left(q_{i}\right)$. Thus we have an embedded surface $\left(\Delta-\bigcup_{i}^{m} h_{i}\left(\left(D^{2}-\{0\}\right) \times \partial I\right)\right) \cup\left(\bigcup_{i}^{m} h_{i}\left(\partial D^{2} \times I\right)\right)$ with the first Betti number $2 m$ and its boundary $K$. This implies that $\Gamma^{*}(K) \leq c^{*}(K)$.

Note that this embedded surface is orientable if and only if the signs of each pair $p_{i}$ and $q_{i}$ are opposite for any $i(=1,2, \ldots, m)$. If $c^{*}(K) \geq 4$, then we can choose a pair of double points in $\Delta$ whose signs are the same. Hence we have $\gamma^{*}(K) \leq c^{*}(K)$ for $c^{*}(K) \geq 4$. On the other hand, if $c^{*}(K)=0$, then by the definition of $\gamma^{*}(K)$, $\gamma^{*}(K)=c^{*}(K)=0$. If $c^{*}(K)=2$, then by Proposition 2.2 and the inequality $\Gamma^{*}(K) \leq c^{*}(K)$, we have $\gamma^{*}(K) \leq \Gamma^{*}(K)+1 \leq c^{*}(K)+1$. 
Next we consider the case that $c^{*}(K)$ is odd. By arguments similar to that in the case above, we have an immersed surface $F$ in a 4 -ball $B^{4}$ with one double point $p$ and $\partial F=K$ such that the first Betti number of the preimage of $F$ is $c^{*}(K)-1$. Take a closed, small neighborhood $N$ of $p$. Note that $\partial(F \cap N)$ is a Hopf link. By removing $F \cap N$ from $F$ and attaching an annulus to it without compatible orientation, we obtain an embedded, nonorientable surface in $B^{4}$ with the first Betti number $c^{*}(K)+1$. Hence we have $\Gamma^{*}(K) \leq \gamma^{*}(K) \leq c^{*}(K)+1$.

From Proposition 2.3 and the definition of $\Gamma^{*}(K)$, we have the following corollary.

Corollary 2.4. For a knot $K$, if $g^{*}(K)=c^{*}(K) \geq 1$, then $\Gamma^{*}(K)=\gamma^{*}(K)$.

Combining Lemmas 1.3 and 1.4 we get the following theorem.

Theorem 2.5. Let $W$ be a compact 4 -manifold with $H_{1}(W ; \mathbb{Z})=0$ and $\partial W \cong S^{3}$. Let $K$ be a knot in $\partial W$ and $D_{K}\left(S^{3}\right)$ the double branched cover of $S^{3}$ with branch set $K$. If $K$ bounds a compact, possibly nonorientale surface $F$ in $W$ that represents zero in $H_{2}\left(W, \partial W ; \mathbb{Z}_{2}\right)$, then the linking form $\lambda$ on $H_{1}\left(D_{K}\left(S^{3}\right) ; \mathbb{Z}\right)$ splits into a direct $\operatorname{sum}\left(G_{1}, \lambda_{1}\right) \oplus\left(G_{2}, \lambda_{2}\right)$ such that

1. there is a $\left(2 \beta_{2}(W)+\beta_{1}(F)\right) \times\left(2 \beta_{2}(W)+\beta_{1}(F)\right)$-matrix $V$ such that $\operatorname{det} V=$ $\pm\left|G_{1}\right|$ and $\lambda_{1}$ is represented by $-V^{-1}$, and

2. $\lambda_{2}$ is metabolic.

From the theorem above, we have the following two corollaries. The second corollary is a '4-dimensional version' of a result of Lickorish [10, Theorem] concerning the nonorientable genus.

Corollary 2.6. Let $K$ be a knot in $S^{3}$ with $\Gamma^{*}(K) \leq n$. Then the linking form $\lambda$ on $H_{1}\left(D_{K}\left(S^{3}\right) ; \mathbb{Z}\right)$ splits into a direct sum $\left(G_{1}, \lambda_{1}\right) \oplus\left(G_{2}, \lambda_{2}\right)$ such that

1. there is an $n \times n$-matrix $V$ such that $\operatorname{det} V= \pm\left|G_{1}\right|$ and $\lambda_{1}$ is represented by $-V^{-1}$, and

2. $\lambda_{2}$ is metabolic.

Corollary 2.7. Let $K$ be a knot in $S^{3}$ with $\Gamma^{*}(K) \leq 1$. Then the linking form $\lambda$ on $H_{1}\left(D_{K}\left(S^{3}\right) ; \mathbb{Z}\right)$ splits into a direct sum $\left(G_{1}, \lambda_{1}\right) \oplus\left(G_{2}, \lambda_{2}\right)$ such that

1. $\lambda_{1}$ is represented by the $1 \times 1$-matrix $\pm\left(1 /\left|G_{1}\right|\right)$, and

2. $\lambda_{2}$ is metabolic.

From the corollary above, we have

Example 2.8. $\Gamma^{*}\left(4_{1}\right)=\gamma^{*}\left(4_{1}\right)=2$.

It is already known that $\gamma^{*}\left(4_{1}\right)=2[17],[18]$.

Proof. Since we can easily see that $\Gamma^{*}\left(4_{1}\right) \leq \gamma^{*}\left(4_{1}\right) \leq 2$, we shall prove that $\Gamma^{*}\left(4_{1}\right) \geq 2$. A Goeritz matrix for $4_{1}$ is

$$
\left(\begin{array}{cc}
-3 & 1 \\
1 & -2
\end{array}\right)
$$

So we see that $H_{1}\left(D_{4_{1}}\left(S^{3}\right) ; \mathbb{Z}\right) \cong \mathbb{Z} / 5 \mathbb{Z}$ and that the linking form $\lambda$ is defined by $\lambda(g, g)= \pm 2 / 5$ for some generator $g$. Note that $\lambda$ has no metabolic part because 5 is square-free. Suppose that $\Gamma^{*}\left(4_{1}\right) \leq 1$. From Corollary 2.7 there is some integer $n$ such that $\lambda(n g, n g)= \pm 1 / 5$. Thus we have $\pm 1 / 5=\lambda(n g, n g)=n^{2} \lambda(g, g)=2 n^{2} / 5$ in $\mathbb{Q} / \mathbb{Z}$. But this is a contradiction since an easy calculation shows this cannot occur. 
The following remark, Remark[2.9 (1), (2), implies that the inequalities in Propositions 2.1 and 2.3 are best possible. Remark 2.9 (3) gives a nontrivial example that implies the inequality $\Gamma^{*}(K) \leq c^{*}(K)$ (for $c^{*}(K)$ is even) in Proposition 2.3] is best possible.

Remark 2.9. (1) For a slice knot $K$, we have $\Gamma^{*}(K)=\gamma^{*}(K)=c^{*}(K)=0$.

(2) Since $\gamma\left(4_{1}\right) \leq 2$ and $c^{*}\left(4_{1}\right) \leq 1$, by Example 2.8 we have $\Gamma^{*}\left(4_{1}\right)=\gamma^{*}\left(4_{1}\right)=$ $\gamma\left(4_{1}\right)=c^{*}\left(4_{1}\right)+1=2$.

(3) For a knot $K, \mathrm{~K}$. Murasugi [1] gave the inequality $|\sigma(K)| / 2 \leq g^{*}(K)$, where $\sigma(K)$ is the signature of $K$. Since $\left|\sigma\left(3_{1} \# 3_{1}\right)\right|=4$ and $g^{*}\left(3_{1} \# 3_{1}\right) \leq c^{*}\left(3_{1} \# 3_{1}\right) \leq 2$, we have $g^{*}\left(3_{1} \# 3_{1}\right)=c^{*}\left(3_{1} \# 3_{1}\right)=2$. In [18] the second-named author showed that $\gamma^{*}\left(3_{1} \# 3_{1}\right) \geq 2$. Hence by Proposition 2.3 and Corollary 2.4 , we have $\gamma^{*}\left(3_{1} \# 3_{1}\right)=$ $\Gamma^{*}\left(3_{1} \# 3_{1}\right)=c^{*}\left(3_{1} \# 3_{1}\right)=2$.

Unfortunately, the authors do not know whether the inequality in Proposition 2.2 is best possible or not. Note that the authors in 12 showed that an inequality $\gamma(K) \leq 2 g(K)+1$ is best possible. So we have the following conjecture.

Conjecture 2.10. There is a knot $K$ such that

$$
\gamma^{*}(K)=2 g^{*}(K)+1 .
$$

\section{REFERENCES}

1. S. Akbulut and R. Kirby, Branched covers of surfaces in 4-manifolds, Math. Ann. 252 (1980), 111-131. MR 82j:57001

2. J.W. Alexander and G.B. Briggs, On types of knotted curves, Ann. of Math. 28 (1927), 562586.

3. G. Burde and H. Zieschang, Knots, de Gruyter Studies in Mathematics, 5. Walter de Gruyter \& Co., Berlin-New York, 1985. MR 87b:57004

4. B.E. Clark, Crosscaps and knots, Internat. J. Math. and Math. Sci. 1 (1978), 113-123. MR 57:17620

5. R.H. Fox, Some problems on knot theory, 1962 Topology of 3-manifolds and related topics (Proc. The Univ. of Georgia Institute, 1961) pp. 168-176 Prentice-Hall, Englewood Cliffs, N.J. MR 25:3523

6. P.M. Gilmer, On slice genus of knots, Invent. Math. 66 (1982), 191-197. MR 83g:57003

7. L. Goeritz, Knoten und quadratische Formen, Math. Z. 36 (1933), 647-654.

8. C.McA. Gordon and R.A. Litherland, On the signature of a link, Invent. Math. 47 (1978), 53-69. MR 58:18407

9. W.B.R. Lickorish, The unknotting number of a classical knot, Combinatorial methods in topology and algebraic geometry (Rochester, N.Y., 1982), 117-121, Contemp. Math., 44, Amer. Math. Soc., Providence, R.I., 1985. MR 87a:57012

10. W.B.R. Lickorish, Unknotting by adding a twisted band, Bull. London Math. Soc. 18 (1986), 613-615. MR 88e:57008

11. K. Murasugi, On a certain numerical invariant of link types, Trans. Amer. Math. Soc. 117 (1965), 387-422. MR 30:1506

12. H. Murakami and A. Yasuhara, Crosscap number of a knot, Pacific J. Math. 171 (1995), 261-273. MR 96k:57006

13. V.A. Rohlin, Two-dimensional submanifolds of four-dimensional manifolds, Functional Anal. Appl. 5 (1974), 39-48. MR 45:7733

14. D. Rolfsen, Knots and links, Mathematics Lecture Series, No. 7. Publish or Perish, Inc., Berkeley, Calif., 1976. MR 58:24236; corrected reprint MR 95c:57018

15. H. Seifert, Die Verschlingungsinvarianten der zyklischen Knotenüberlagerungen, Abh. Math. Sem. Univ. Hamburg 11 (1935), 84-101.

16. T. Shibuya, Some relation among various numerical invariants for links, Osaka J. Math. 11 (1974), 313-322. MR 50:5779 
17. O.Ya. Viro, Positioning in codimension 2, and the boundary. (Russian) Uspehi Mat. Nauk 30 (1975), 231-232. MR 54:8654

18. A. Yasuhara, Connecting Lemmas and representing homology classes of simply connected 4-manifolds, Tokyo J. Math. 19 (1996), 245-261. MR 97g:57027]

Department of Mathematics, School of Science and Engineering, Waseda University, Ohkubo 3-4-1, Shinjuku-ku, Tokyo 169-8555, Japan

E-mail address: starshea@tky3.3web.ne.jp

Department of Mathematics, Tokyo Gakugei University, Nukuikita 4-1-1, Koganei, TOKYO 184-8501, JAPAN

E-mail address: yasuhara@u-gakugei.ac.jp

Current address, October 1, 1999 to September 30, 2001: Department of Mathematics, The George Washington University, Washington, DC 20052

E-mail address: yasuhara@research.circ.gwu.edu 